PEDIATRIC UROLOGY

doi: $10.1590 / S 1677-55382011000200030$

\title{
Risk factors for progression to end-stage renal disease in children with posterior urethral valves
}

Ansari MS, Gulia A, Srivastava A, Kapoor R

Department of Urology and Renal Transplantation, Sanjay Gandhi Postgraduate Institute of Medical Sciences, Lucknow, India

J Pediatr Urol. 2010; 6: 261-4

Objective: To identify the variables which affect long-term renal outcome in children with posterior urethral valves (PUV).

Materials and Methods: Retrospective analysis of 260 children with PUV who underwent ablation of valves in 1992-2008 at our tertiary care center. The following risk factors for progression to end-stage renal disease (ESRD) were analyzed: nadir serum creatinine greater than $1.0 \mathrm{mg} / \mathrm{dL}$, bilateral grade 3 or higher VUR at diagnosis, recurrent febrile UTIs, and severe bladder dysfunction. Patients were divided into two groups: those who developed ESRD (group 1) and those who did not (group 2).

Results: Forty (17.62\%) patients had nadir serum creatinine $>1 \mathrm{mg} / \mathrm{dL}$. At time of initial presentation, highgrade VUR was seen in $63.1 \%$ and $33.5 \%$ of groups 1 and 2 , respectively $(\mathrm{P}=0.002)$. Overall, $77(34 \%)$ of the boys developed breakthrough urinary tract infections: $37.03 \%$ and $33.5 \%$ in groups 1 and 2 , respectively $(\mathrm{P}=$ 1). Fifty-nine (26\%) patients were found to have severe bladder dysfunction: $77.8 \%$ and $19 \%$ in groups 1 and 2 , respectively $(\mathrm{P}<0.0001)$. Twenty-seven $(11.89 \%)$ patients progressed to ESRD, at mean age of 11.21 years (5-16). On univariate analysis, the risk-predicting variables were: nadir serum creatinine value greater than $1 \mathrm{mg} / \mathrm{dL}(\mathrm{P}<0.0001)$, bilateral high-grade VUR $(\mathrm{P}=0.002)$ and severe bladder dysfunction $(\mathrm{P}<0.0001)$. On multivariate logistic regression analysis, nadir serum creatinine greater than $1 \mathrm{mg} / \mathrm{dL}$ (OR 23.79; CI 8.20-69.05) and severe bladder dysfunction (OR 5.67; CI 1.90-16.93) were found to be independent risk factors predictive of ultimate progression to ESRD.

Conclusions: Nadir serum creatinine and bladder dysfunction are the main factors affecting long-term renal outcome in cases of PUV. Early identification and treatment of bladder dysfunction may thus be beneficial.

\section{Editorial Comment}

This paper looked at a large series of children with posterior urethral valves in order to identify risk factors for end-stage renal disease. Patients were followed for a mean of 7.2 years. A total of 227 patients were eligible for inclusion in the review. The authors looked at age at presentation, nadir serum creatinine, presence of high-grade reflux, recurrent febrile urinary tract infections, and severe bladder dysfunction as variables. 30\% of patients developed chronic kidney disease and $12 \%$ progressed to end-stage renal disease. On univariate analysis nadir creatinine greater than $1 \mathrm{mg} / \mathrm{dL}$, bilateral high-grade reflux, and severe bladder dysfunction were found to be risk factors. On multivariate analysis; however, only nadir serum creatinine greater than $1 \mathrm{mg} / \mathrm{dL}$ and severe bladder dysfunction were predictive of progression to end-stage renal disease.

The strength of this study is the large number of patients at a single institution. Certainly longer follow-up would likely reveal a greater number of patients who progress to end-stage renal disease. Their findings are in line with another large series recently published which also demonstrated bladder dysfunction and nadir creatinine to be the only independent risk factors for end-stage renal disease using multivariate analysis (1). While nadir serum creatinine is not a modifiable risk factor, bladder dysfunction can certainly be managed aggressively in these at risk patients. This paper helps to reemphasize the importance of screening for bladder 
dysfunction early on. It remains to be seen whether or not early and aggressive management of bladder dysfunction can have an impact on outcomes.

\section{Reference}

1. DeFoor W, Clark C, Jackson E, Reddy P, Minevich E, Sheldon C: Risk factors for end stage renal disease in children with posterior urethral valves. J Urol. 2008; 180(4 Suppl): 1705-8; discussion 1708.

Dr. M. Chad Wallis

Division of Pediatric Urology

University of Utah

Salt Lake City, Utah, USA

E-mail: chad.wallis@hsc.utah.edu

doi: 10.1590/S1677-55382011000200031

Safety of shock wave lithotripsy for treatment of pediatric urolithiasis: 20-year experience

Griffin SJ, Margaryan M, Archambaud F, Sergent-Alaoui A, Lottmann HB

Pediatric Surgery Service, Necker Hospital for Sick Children, Paris, France

J Urol. 2010; 183: 2332-6

Purpose: This retrospective study was designed to assess the impact of shock wave lithotripsy on the pediatric kidney using pretreatment and posttreatment $(99 \mathrm{~m})$ technetium dimercapto-succinic acid renal scintigram.

Materials and Methods: A total of 182 patients 5 months to 19.8 years old (mean 5.3 years) were treated for renal calculi with shock wave lithotripsy during a 20 -year period. Pretreatment evaluation included clinical assessment, urine culture, renal ultrasound and plain abdominal radiograph with or without excretory urogram. Dimercapto-succinic acid scintigram was performed before and 6 months after completion of treatment in 94 patients (52\%).

Results: Patients underwent 1 to 4 sessions of shock wave lithotripsy per kidney with at least 1 month between treatments. Median number of shocks delivered per session was 3,000 (IQR 2,601 to 3,005). No new scars were observed on any posttreatment dimercapto-succinic acid scan. Regarding renal function, patients fell into 1 of 4 groups. Group 1 (66 patients, 70\%) had normal function on dimercapto-succinic acid scan before and after treatment, group $2(18,19 \%)$ had decreased function in the affected kidney on pretreatment scan with no change after treatment, group $3(2,2 \%)$ had impaired function in the treated kidney that was transient (1) or permanent (1) and group 4 (7, 7\%) had improved function in the treated kidney.

Conclusions: Shock wave lithotripsy is an effective treatment for renal calculi in children. Renal parenchymal trauma associated with extracorporeal shock wave lithotripsy does not seem to cause long-term alterations in renal function or development of permanent renal scars in children.

\section{Editorial Comment}

This retrospective study looked at 182 pediatric patients who were treated for renal stones over 20 years using shock wave lithotripsy. They had a low number of complications with steinstrasse developing in 2 patients and another 2 patients who developed pyelonephritis. Part of their preoperative assessment included a DMSA scan. What is remarkable about this study is that they were able to get posttreatment DMSA scans in 94 patients six months following lithotripsy. No new renal parenchyma scars were discovered on their follow- 